BMJ Open

Sport \&

Exercise

Medicine

\title{
Preventing lower extremity injury in elite orienteerers: study protocol for a randomised controlled trial
}

\author{
Philip von Rosen, Bodil Halvarsson
}

To cite: von Rosen $P$, Halvarsson B. Preventing lower extremity injury in elite orienteerers: study protocol for a randomised controlled trial. BMJ Open Sport \& Exercise Medicine 2018;4:e000347. doi:10.1136/ bmjsem-2018-000347

Accepted 30 March 2018
Check for updates

Department of Neurobiology, Care Sciences, and Society (NVS) Division of Physiotherapy, Karolinska Institutet, Huddinge, Sweden

\section{Correspondence to}

Dr Philip von Rosen, Division of Physiotherapy, NVS, Karolinska Institutet, Huddinge 14183 , Sweden; philip.von.rosen@ki.se

\section{ABSTRACT}

Background The high physical load associated with running through uneven terrain contributes to orienteerers being exposed to high injury risk, where the majority of injuries are located in the lower extremities. Specific training programmes have been effective at reducing injury risk in sports. Yet no trial has been conducted in elite orienteering. The aim of this study is to investigate the effectiveness of a specific training programme in preventing lower extremity injury in adult elite orienteerers. Study design Randomised controlled trial (RCT). Methods Seventy-two Swedish elite orienteerers, aged 18-40 years, are allocated to an intervention or control group. The intervention group performs four specific exercises, with three difficult levels intensified every second week over the first 4 weeks, targeting strength, flexibility and coordination of the lower extremity. The exercises are completed four times a week ( $10 \mathrm{~min}$ per session) in conjunction with normal training over 14 weeks. Injury data are collected every second week using a valid injury questionnaire distributed by text messages over 14 weeks. The primary outcome is number of substantial injuries in the lower extremity. The secondary outcomes are incidence of ankle sprains and the average substantial injury prevalence across 14 weeks.

Discussion Due to high injury risk and lack of injury prevention trials in orienteering, an RCT investigating the effect of a specific exercise programme in preventing lower extremity injury is warranted. The results of this trial will be beneficial to orienteerers, clubs and federations, and increase our understanding on how lower extremity injuries can be prevented in a physically challenging sport. Trial registration number NCT03408925.

\section{Introduction}

In orienteering, orienteerers run through tough terrain and at the same time make route choices in order to complete the course as fast as possible. The high physical load associated with running through uneven terrain contributes to orienteerers being exposed to high injury risk. ${ }^{1}$ Across a season more than $8 \%$ of young elite orienteerers will report to be substantially injured each week. ${ }^{2}$ Most injuries seem to occur during competitions, where the injury incidence is reported to be 7-15/1000 competition hours compared with 2-3/1000 training hours. Ekstrand $e t a \hat{l}^{3}$ registered injuries during a 5-day competition and found the incidence rate to be 7 injuries per 1000 competition hours. However, even if injury risk is high during competitions, few prospective injury registration studies following orienteerers across a season are available. $^{245}$

The majority of injuries in orienteering are located in the lower extremities, ${ }^{5-8}$ with $70 \%-94 \%$ of all injuries located in this body region. ${ }^{46-8}$ Ankle sprains appear to be one of the most frequent injuries. ${ }^{3-5} 7910$ However, the risk factors for ankle sprains or other lower extremity injuries in orienteering are limitedly explored, with few longitudinal studies available. In the few published studies, history of injury, current injury at study start, a high training volume, long competition time and running on asphalt and paths have been identified as injury risk factors in adolescent elite orienteerers. ${ }^{24}$ Yet no trial has been conducted aiming to prevent injuries in elite orienteerers.

In a general sporting population, proprioceptive training programmes are effective at reducing ankle sprains. ${ }^{11}$ However, orienteerers are also exposed to other serious injuries in the lower extremity, such as medial shin pain, Achilles peritendinitis and iliotibial band friction syndrome, ${ }^{5}$ indicating that a different approach is warranted in order to effectively prevent injuries in orienteering. Besides, orienteerers' high stress exposure due to running on uneven terrain may also suggest that successful training programmes in team sports may be less effective in this population, ${ }^{1}$ which calls for sport-specific training interventions.

In summary, the high injury risk of lower extremity injury in elite orienteerers indicates a need for preventive actions, ${ }^{245}$ addressing multiple causes of injuries in this population. ${ }^{12} 13$ The purpose of this study protocol is to present the study design of a randomised controlled trial (RCT) aiming to explore the effectiveness of a specific training programme 
in preventing lower extremity injury in adult elite orienteerers.

\section{METHODS AND ANALYSIS Design and randomisation}

This is a prospective RCT investigating the effect of performing an exercise programme on preventing lower extremity injury. The trial is designed in accordance with the Consolidated Standards of Reporting Trials, developed to alleviate the problems arising from inadequate reporting of RCTs. It is hypothesised that a specific exercise programme can reduce the number of lower extremity injuries, including both acute and overuse injuries, in adult female and male elite orienteerers with a medium effect size. A computer-generated stratified random assignment (by sex) will be used to randomise orienteerers to the control or exercise group.

\section{Study setting}

This trial is being carried out in collaboration with the National Federation of Orienteering. Adult elite orienteerers will be enrolled at four sites in Sweden. Each of the four sites has organised training facilities for elite male and female orienteerers and thereby attracts a high number of elite orienteerers yearly. To get in contact with the orienteerers, the coaches at each site will be approached. In case of recruitment difficulties, the National Federation of Orienteering will be approached to allow for direct contact with suitable orienteerers.

\section{Eligibility criteria}

Swedish adult elite orienteerers, aged 18-40 years, are eligible for inclusion. Elite orienteerer is defined as an orienteerer participating in at least one competition in the highest national orienteering league (Silva League). Athletes not able to complete the initial screening tests (heel-rise test, square hop test) or have not been able to run the last week due to injury will be excluded.

\section{Data collection}

Orienteerers who agree to participate in the study will be invited to a meeting and then orally informed of the purpose of the study and the voluntary nature of participation. To be included in the study, all participants need to fulfil the inclusion and exclusion criteria and provide written informed consent. The included orienteerers will then be asked to complete a baseline questionnaire about demographics (eg, age, height, weight), training history including relevant medical history, as well as the Hassles and Uplifts Scale and the Self-Perceived Stress Scale-14 (PSS-14). ${ }^{14} 15$ The orienteerers will also be asked to perform the heel-rise test and the square hop test (described below). Randomisation will be completed after the baseline data have been collected, directly followed by the 14-week intervention period.

Injury data will be collected using the valid version of the Oslo Sports Trauma Research Center (OSTRC) Overuse Injury Questionnaire. ${ }^{16}$ The injury questionnaire will
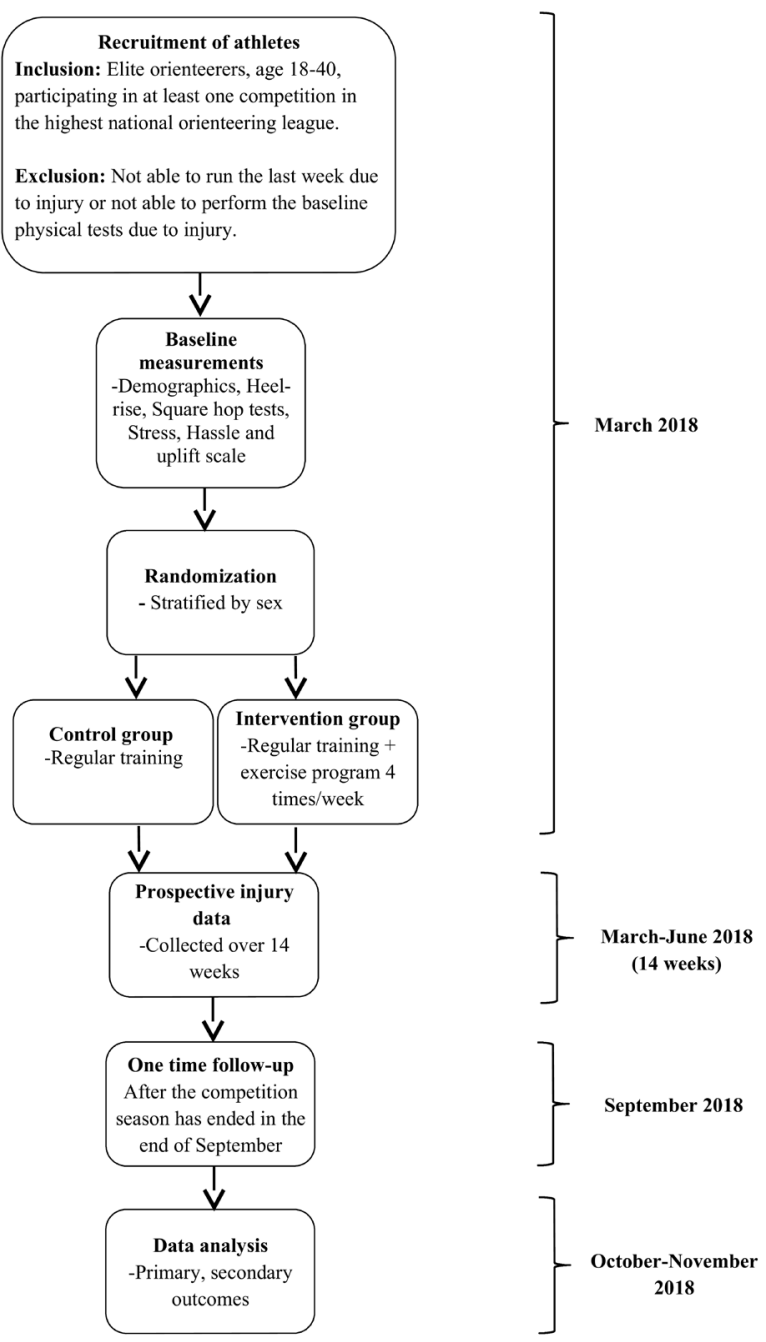

Figure 1 Flow chart of participant enrolment.

be provided to the participating orienteerers using text messages delivered each second week. In case of non-response, a reminder will be sent 4 days later. If still no response, the orienteerer will be interviewed by phone the next week. The athletes will be continuously followed over 14 weeks, including both preseason and competitive season (figure 1). A further one-time follow-up is planned after the competitive season has ended (end of September), where the participants will be asked to complete a similar injury questionnaire as before. Test assessors and data collectors will be blinded to treatment allocation. The randomised phase will be unblinded after 14 weeks of data collection. Long-term follow-up (4 months) will be conducted by test assessors blinded to treatment allocation.

\section{Injury questionnaire}

The OSTRC Overuse Injury Questionnaire measures injury consequences on sports participation, performance, training and pain based on four questions with alternative responses. It assesses the effect of injuries on participation (four responses ranging from 'full 
participation' to 'cannot participate'), reduction in training volume (five responses ranging from 'no reduction' to 'cannot participate'), reduced sporting performance (five responses ranging from 'no effect' to 'can not participate') and experience of pain (four responses ranging from 'no pain' to 'severe pain'). Substantial injury data will be extracted, where substantial injury is defined as 'any physical complaint resulting in moderate or severe reductions in training volume, or moderate or severe reduction in performance, or complete inability to participate in sports'. The OSTRC Overuse Injury Questionnaire has been shown to have acceptable content and construct validity with a Cronbach's alpha of $0.91 .{ }^{1617}$

The OSTRC Overuse Injury Questionnaire will be adjusted to orienteering by including questions about training volume, occurrence of ankle sprains and how often the exercise programme has been completed. Every second distributed questionnaire also includes the PSS-14.

\section{Screening tests}

\section{Heel-rise test}

The subject will be instructed to stand barefoot on a $10^{\circ}$ tilted wedge and asked to perform a maximal number of single-leg heel rises with a straight knee and trunk. Before the test, the maximal height of a single heel rise at each leg is measured with a ruler as the normal heel rise height. The subject is then instructed to repeatedly lift the heel as high and return to start position, until no more heel lifts could be performed. If the subject did not reach the normal heel rise height measured before the test, a verbal feedback will be given and that specific heel rise will not be counted. After two verbal feedback, the test will be over. The number of correct heel rises will be counted. To assist balance, individuals are permitted to apply fingertip support. A metronome will be used to control heel-rise cycle at 60 per minute. The right leg is tested before the left leg. The test has shown excellent test-retest reliability $(=1.0) .{ }^{18}$

\section{Square hop test}

The subject stands on the right leg with the hand behind their back, outside a $30 \times 35 \mathrm{~cm}$ square marked with tape on the floor. The subject is then asked to complete as many jumps as possible in all four directions, in a clockwise in and out procedure, during a period of $30 \mathrm{~s}$. Touching the tape is recorded as an error and not as a correct jump. For the left leg, the subject performs the test in a counter-clockwise mode. Inter-reliability and intrareliability have been found to be satisfactory $(\mathrm{r}=0.79-0.94) .^{19}$

\section{Stress}

The PSS-14 addresses general feelings and thoughts about unpredictable and uncontrollable life events and to what extent these situations are appraised as stressful. It consists of 14 items and questions are answered on a 5-grade Likert-type scale from 0 (never) to 4 (very often), where a high total score indicates a high level of self-perceived stress. The PSS scale has been found to have good internal reliability, as well as satisfactory construct, criterion and predictive validity. ${ }^{2021}$

\section{The Hassles and Uplift Scale}

The Hassles and Uplifts Scale measures an athlete's level of daily hassles and uplifts. It consists of 53 items addressing potential daily hassles and uplifts such as family issues, health, financial issues and so on. The athlete is asked how much of a hassle and how much of an uplift each item has been the last week. Questions are answered on a 4-grade Likert-type scale ranging from 0 (not at all) to 3 (very much). In a cohort of athletes from different sports, the Cronbach's alpha was $0.82 .{ }^{22}$

\section{Intervention}

The intervention consists of an exercise programme developed by the medical team of the National Federation of Orienteering. Specifically, it consists of four exercises targeting strength, flexibility and coordination of the lower extremity. The orienteerers are asked to perform the exercises four times a week throughout the entire study period. The exercises are one-leg stance, runner's pose, one leg heel raise and one leg side hop with three difficulty levels aiming to mainly improve lower extremity strength and neuromuscular function (figure 2). Every second week the exercises' difficulty level will be increased. Consequently, the orienteerers will be on the highest level of difficulty after 4 weeks and then asked to continue at that level throughout the study. To perform the exercises as correctly as possible, the orienteerers will be provided pictures of the exercises as well as verbal explanation on exercise performance quality. The first level of the four exercises will be explained and demonstrated to the orienteerers after the randomisation process.

\section{Data analysis}

Based on previous studies, ${ }^{24}$ we estimate the effect size between the intervention and the control groups for the number of substantial injuries in the lower extremity (primary outcome), allowing for multiple injuries per individual including both acute and overuse injuries to be 0.3 . With two-sided testing, a significance level of 0.05 and a power of 0.8 , each study group is estimated to include 33 athletes. Due to dropouts $(n=6)$, this study aims to include 72 elite orienteerers. The secondary outcomes are incidence of ankle sprains and the average substantial injury prevalence across the 14 weeks.

Multiple imputation techniques will be used to handle missing data. Descriptive statistics (means and SD) will be used to describe baseline characteristics and exposure data. t-Tests and Mann-Whitney $\mathrm{U}$ tests will be used for continuous variables and $\chi^{2}$ tests for categorical variables. Data analysis will be based on an intention-to-treat 


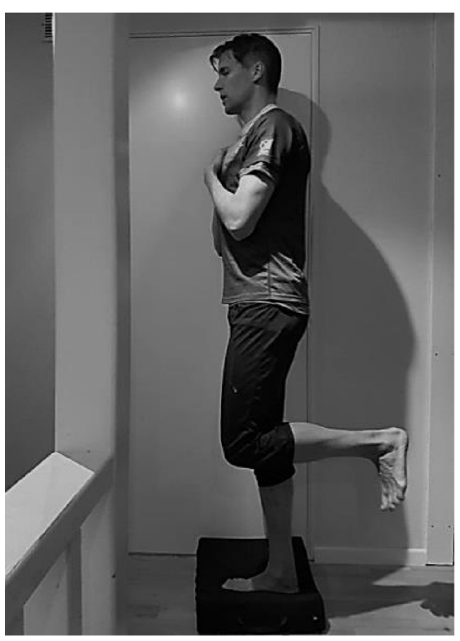

A)

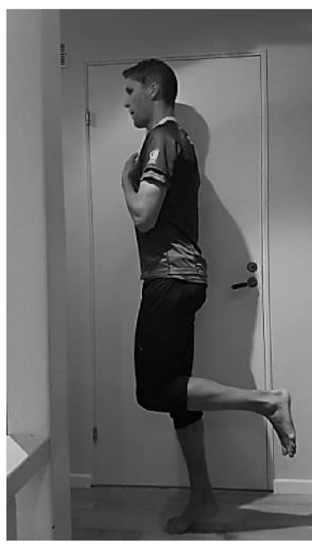

C)
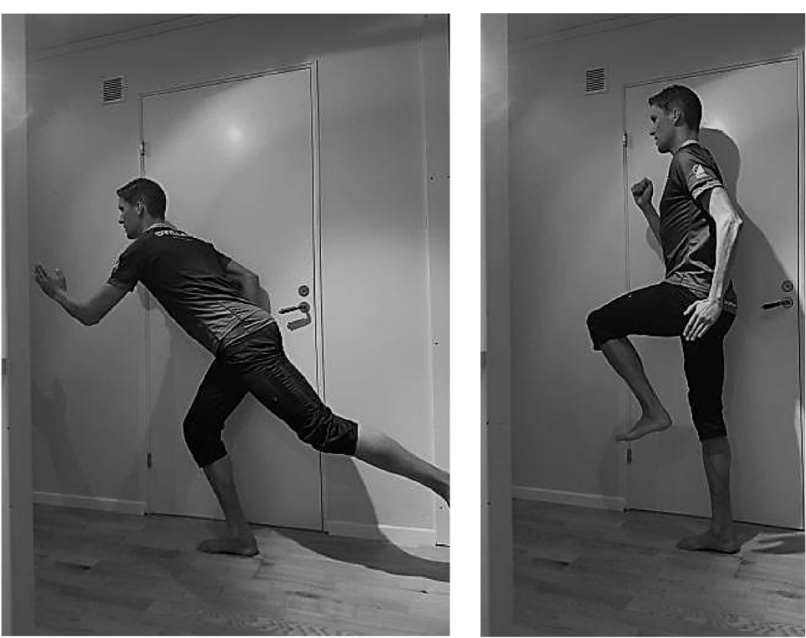

B)

\section{政}

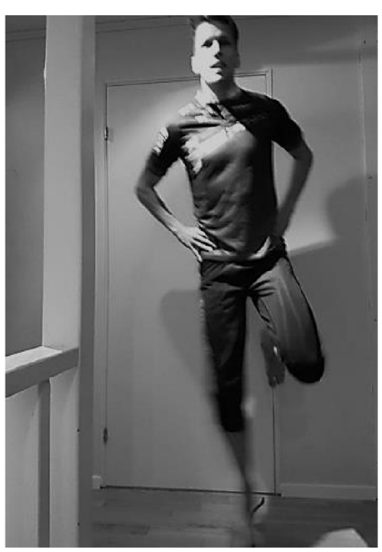

D)

Figure 2 One-leg stance (A), 2 min/leg. Level 1, one-leg stance with eyes closed on soft surface; level 2, one-leg stance with eyes closed on soft surface with arm/leg movements; level 3, one-leg stance with eyes closed on soft surface repeatedly performing single leg knee bends. Runner's pose (B), 3×10/leg. Level 1, single leg stance simulating a runner's step; level 2 , single leg stance simulating a runner's step with a one leg heel raise in end position; level 3, single leg stance simulating a runner's step, on a soft surface, with a one leg heel raise in end position. One leg heel raise (C), $3 \times 15 /$ leg. Level 1 , one leg heel raise without finger support; level 2, one leg heel raise in a stair with finger support; level 3, one leg heel raise in a stair without finger support. One leg side hop (D), 2×30/leg. Level 1, one leg side hop, $40 \mathrm{~cm}$ distance, optional arm position; level 2, one leg side hop, $40 \mathrm{~cm}$ distance, with crossed arms; level 3, one leg side hop, $40 \mathrm{~cm}$ distance, with crossed arms and simultaneous cervical rotation.

basis and analysed separately for the competitive season and the complete season (preseason and competitive season) using mixed effects logistic regression. Subgroup analysis will compare orienteerers with a high compliance with those athletes with a low compliance. Analyses will separately consider multiple injuries as well as the proportion of injured population. In secondary analyses, Poisson general log-linear analysis and Cox hazards regression with survival curves will be used to analyse risk factors for substantial injury in the lower extremity with baseline data and screening test data as covariates. All analyses were performed using the SPSS software for Windows (V.24.0), with the level of significance set at a $p$ value $\leq 0.05$ for all tests.

\section{ETHICS AND DISSEMINATION Participant safety considerations}

This RCT will be conducted in cooperation with and under the supervision of medical personnel from the Swedish National Federation of Orienteering. In case of severe injury or illness, the injured orienteerer will be referred to medical personnel. The intervention programme has previously been tested by the National Federation of Orienteering and is considered to decrease and not enlarge the injury risk.

\section{Ethical considerations}

This study will be conducted in compliance with the Declaration of Helsinki. All participants will receive 
oral and written information about the study and sign a written consent to participate. The study has been registered at the US National Library of Medicine ClinicalTrials.gov database (NCT03408925).

\section{Dissemination plan}

The final report will be submitted to peer-reviewed journals for publication in English at the end of 2018. Study data will be deposited in safe, long-term storage at Karolinska Institutet, according to the Local Ethics Committee of Stockholm.

\section{DISCUSSION}

Orienteerers are at high risk for lower extremity injuries, ${ }^{245}$ which yearly lead to missed sport participation, reduced performance and personal suffering. So far, no trial has been conducted to reduce the high number of substantial injuries yearly affecting this population. Based on results from other sports, it is possible to prevent injuries in the lower extremity, such as ankle sprains. ${ }^{11}$ However, due to completely different physical load on orienteerers, ${ }^{1}$ data from team sports athletes cannot be extrapolated to elite orienteerers. This prompted the authors to conduct an RCT exploring the effectiveness of a specific training programme in adult elite orienteerers. The prevention of substantial injuries will be beneficial to orienteerers, clubs and federations, and provide data on how lower extremity injuries can be prevented. We believe our results can be extended to adolescent and adult elite and subelite orienteerers and serve as a foundation to develop injury prevention programme in recreational orienteerers.

This study has the advantage of including a homogeneous group of elite orienteerers followed during both preseason and competitive season. Modern injury definition suited for self-reported data will be applied. Besides the main outcome, data on stress and physical screening tests will also be collected, providing an opportunity of exploring several risk factors for injuries in adult elite orienteerers. In summary, due to high injury risk and lack of injury prevention trials in orienteering, a new perspective on injury risk and prevention in this sport is warranted. The planned RCT aims at providing all of that in elite orienteering.

\section{TRIAL STATUS}

Participants will be recruited in March 2018. The intervention starts in March 2018 and data collection will be completed in June 2018. Data analyses are expected to be completed in October-November 2018.

Contributors PvR and BH initiated the overall project and designed the study. PvR prepared the first draft of the manuscript. Both authors made substantial contributions to the revision of the document prior to submission.

Funding This research is funded by the Swedish National Federation of Orienteering. PvR and BH report no additional grants to conduct this study.

Competing interests None declared.
Patient consent Not required.

Ethics approval Regional Ethical Committee in Stockholm, Sweden (Dnr 2018/135-32).

Provenance and peer review Not commissioned; externally peer reviewed.

Open Access This is an Open Access article distributed in accordance with the Creative Commons Attribution Non Commercial (CC BY-NC 4.0) license, which permits others to distribute, remix, adapt, build upon this work non-commercially, and license their derivative works on different terms, provided the original work is properly cited and the use is non-commercial. See: http://creativecommons.org/ licenses/by-nc/4.0/

(C) Article author(s) (or their employer(s) unless otherwise stated in the text of the article) 2018. All rights reserved. No commercial use is permitted unless otherwise expressly granted.

\section{REFERENCES}

1. Creagh U, Reilly T. Physiological and biomechanical aspects of orienteering. Sports Med 1997;24:409-18.

2. von Rosen P, Floström F, Frohm A, et al. Injury patterns in adolescent elite endurance athletes participating in running, orienteering, and cross-country skiing. Int J Sports Phys Ther 2017;12:822-32.

3. Ekstrand J, Roos H, Tropp H. The incidence of ankle sprains in orienteering. Sci J Orienteering 1990;6:3-9.

4. von Rosen P, Heijne Al, Frohm A. Injuries and associated risk factors among adolescent elite orienteerers: a 26-week prospective registration study. J Athl Train 2016;51:321-8.

5. Linde F. Injuries in orienteering. Br J Sports Med 1986;20:125-7.

6. Johansson C. Injuries in elite orienteers. Am J Sports Med 1986;14:410-5.

7. Linko PE, Blomberg HK, Frilander HM. Orienteering competition injuries: injuries incurred in the Finnish Jukola and Venla relay competitions. Br J Sports Med 1997;31:205-8.

8. Roos L, Taube W, Zuest P. Musculoskeletal injuries and training patterns in junior elite orienteering athleletal Injuries and Training Patterns in Junior Elite Orienteering Athletes. Biomed Res Int 2015;2015:259531-8.

9. Hintermann B, Hintermann M. Injuries in orienteering. A Study of the 1991 Swiss 6-days orienteering event. Sci J Orienteering 1992;8:72-8.

10. Mclean I. First aid for orienteering in Scotland. Sci J Orienteering 1990;6:55-63.

11. Schiftan GS, Ross LA, Hahne AJ. The effectiveness of proprioceptive training in preventing ankle sprains in sporting populations: a systematic review and meta-analysis. J Sci Med Sport 2015;18:238-44.

12. Bahr R, Holme I. Risk factors for sports injuries-a methodological approach. Br J Sports Med 2003;37:384-92.

13. Meeuwisse $\mathrm{WH}$, Tyreman $\mathrm{H}$, Hagel $\mathrm{B}$, et al. A dynamic model of etiology in sport injury: the recursive nature of risk and causation. Clin J Sport Med 2007;17:215-9.

14. Cohen S, Kamarck T, Mermelstein R. A global measure of perceived stress. J Health Soc Behav 1983;24:385-96.

15. DeLongis A, Folkman S, Lazarus RS. The impact of daily stress on health and mood: psychological and social resources as mediators. J Pers Soc Psychol 1988;54:486-95.

16. Clarsen B, Myklebust G, Bahr R. Development and validation of a new method for the registration of overuse injuries in sports injury epidemiology: the Oslo Sports Trauma Research Centre (OSTRC) overuse injury questionnaire. Br J Sports Med 2013;47:495-502.

17. Ekman E, Frohm A, Ek P, et al. Swedish translation and validation of a web-based questionnaire for registration of overuse problems. Scand J Med Sci Sports 2015;25:104-9.

18. Hébert-Losier K, Wessman $\mathrm{C}$, Alricsson $\mathrm{M}$, et al. Updated reliability and normative values for the standing heel-rise test in healthy adults. Physiotherapy 2017;103:446-52.

19. Ostenberg A, Roos E, Ekdahl C, et al. Isokinetic knee extensor strength and functional performance in healthy female soccer players. Scand J Med Sci Sports 1998;8(Pt 1):257-64.

20. Nordin M, Nordin S. Psychometric evaluation and normative data of the Swedish version of the 10-item perceived stress scale. Scand J Psychol 2013;54:502-7.

21. Eklund M, Bäckström M, Tuvesson H. Psychometric properties and factor structure of the Swedish version of the Perceived Stress Scale. Nord J Psychiatry 2014;68:494-9.

22. Fawkner HJ, McMurrary NE, Summers JJ. Athletic injury and minor life events: a prospective study. J Sci Med Sport 1999;2:117-24. 